\title{
Design of Phase Shift Control For Transmit/ Receive Module of ST Radar Samikshya Ghosalkar ${ }^{1}$, K.P. Ray ${ }^{1}$, Rajesh Rangari ${ }^{1}$, Saylee Gharge ${ }^{2}$ \\ ${ }^{1}$ SAMEER, IIT Bombay Campus, Powai - 400076. \\ ${ }^{2}$ V.E.S. Institute of Technology, Chembur, Mumbai - 400074. \\ ghosalkar.samikshya@gmail.com, kpray@sameer.gov.in, rangari@sameer.gov.in \\ Sayleegharge73@yahoo.co.in
}

\section{ABSTRACT}

The paper mainly focuses on the beam forming and its analysis for planar phased-array antenna, which is being developed as part of the ST Radar. The beam steering is done electronically by controlling the phase of the T/R module in such a way that the phase difference between each module is equal and it corresponds to the steering angle. The relative phase shift of each T/R module for a specified beam position is worked out using MATLAB and implemented using 6 - bit digital phase shifter. The analytical results are practically demonstrated using the STM32F4xx kit of STMicroelectronics. Theoretical values of the elemental phase for each antenna element in the array, are calculated for various positions of the beam $(\theta \& \varphi)$. The 6 -bit phase shifter results in $5.625^{\circ}$ phase resolution. In the second part of the work, the calculation of the element phase with 6-bit digital phase shifter is discussed. The calculated values of the elemental phase are truncated to an integral multiple of $5.625^{\circ}$.

\section{Indexing terms/Keywords}

Electronic Beam Steering; Transmit Receive Module (TRM); Stratospheric Tropospheric (ST) Radar.

\section{Council for Innovative Research}

Peer Review Research Publishing System

\section{Journal: INTERNATIONAL JOURNAL OF COMPUTERS \& TECHNOLOGY}

Vol 12 , No. 4

editor@cirworld.com 


\section{INTRODUCTION}

SAMEER is developing ST Radar for weather studies. The frequency range of this radar is (195-215) $\mathrm{MHz}$ with $5 \mathrm{MHz}$ bandwidth. The proposed radar shall have an antenna array with 576 elements. Each antenna element will have its own T/R module with a built-in T/R controller. The radar beam can be placed anywhere in azimuth from $0^{\circ}$ to $360^{\circ}$ and $\pm 15^{0}$ in elevation thus forming a cone both in elevation and azimuth angles in steps of $3^{0}$.Each TRM generates $400 \mathrm{~W}$ RF peak power, which results in $230 \mathrm{~kW}$ peak power radiated from the radar. The radar is expected to cover a height range upto 20 $\mathrm{kms}$. For this purpose, an electronic beam steering concept is used.

To steer the beam in the desired direction, the relative phase of the individual elements in the array is adjusted such that the beam points in the desired direction [1-4]. To achieve this, a 6- bit digital phase shifter is included in the T/R module. The use of 6 -bit phase shifter results in $5.625^{\circ}$ resolution. By controlling the phase- shifter insertion phase, the desired phase difference between successive antenna elements is maintained, which results in the beam being pointed in the specific direction. The main objective of the beam steering system is to calculate the elemental phase of the individual elements.

\section{BASICS OF BEAM POSITIONING}

In an antenna array, the beam position is defined by two co-ordinates : elevation angle $(\theta)$ \& azimuth angle $(\phi)$. For a rectangular lattice the $m n^{\text {th }}$ element is located at $X_{m}=m^{*} d_{x}$ and $Y=n^{*} d_{y}$ from where $d_{x}$ and $d_{y}$ are the inter-element distance in $X$ and $Y$ direction respectively. The distribution of the antenna elements in the antenna array is shown in Fig.1 below:

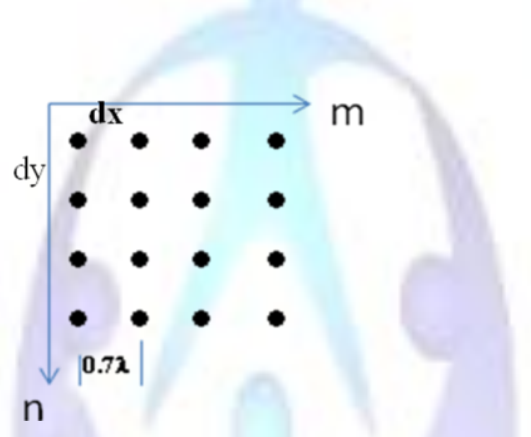

Fig.1 Distribution of Antenna Elements.

To steer the beam in any direction $(\theta, \varphi)$ in space, the phase data for each element is calculated as Element Phase $=m * \delta x+n * \delta y$

Where $\delta \mathrm{x}$ is the phase gradient in $\mathrm{X}$-direction and $\delta \mathrm{y}$ is the phase gradient in $\mathrm{Y}$-direction.

$\delta x=(2 \pi / \lambda) * d_{x}^{*} \operatorname{Sin}(\vartheta) * \cos (\varphi)$

Where $d_{x}$ is the inter- element spacing $=0.7 \lambda$ which is shown in Fig. 1 and,values of $\theta, \phi$ are taken in degrees, therefore, $2 \pi$ is replaced with $360^{\circ}$.

$\delta x=360 * 0.7 * \sin (\theta) * \cos (\varphi)$

$\delta y=(2 \pi / \lambda) * d_{y} * \operatorname{Sin}(\theta) * \operatorname{Sin}(\phi)$

where, $d_{y}$ is the inter- element spacing $=0.7 \lambda$

$\delta y=360 * 0.7 * \sin (\theta) * \operatorname{Sin}(\varphi)$

For a specific beam position, the values of $\delta x$ and $\delta y$ are constant for all the elements. Thus the relative phase shift depends only on the location $(m, n)$ of the element in the array.

\section{CALCULATION OF ELEMENT PHASE}

Antenna elements in the array are arranged in a square grid configuration with approximately circular aperture. The inter element spacing is $0.7 \lambda$, which results in 28 antenna elements along the diameter. The Fig. 2 below shows the placement of antenna elements in the array. 


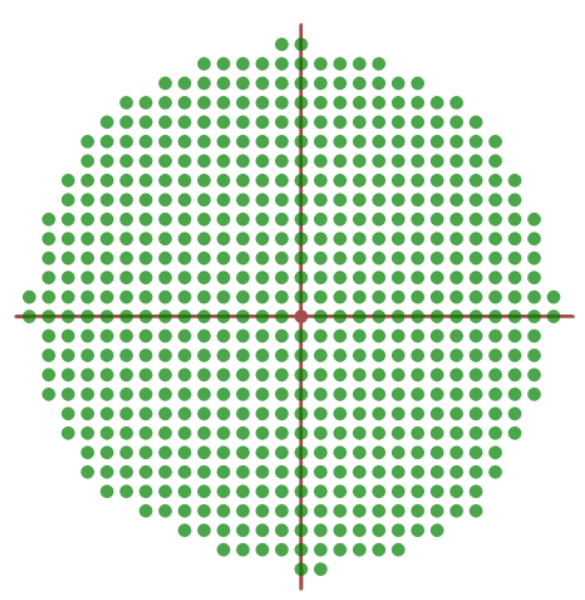

ISSN 2277- 3061

Fig. 2 Antenna Array.

For calculating the element phase of individual element, the reference element $(m, n)=(0,0)$, is taken at the origin of the circle. Thus, both $m$ and $n$ vary from -14 to +13 along the center line. All the calculations of element phase have been carried out as per this definition of $m \& n$. For proper beam formation, the phase shift of each of the 576 elements should be appropriate. Each TRM has to generate phase shift for the antenna associated with it for azimuth angle variation of $(0-$ $360)^{0}$ and elevation angle $(0-15)^{0}$. The beam is steered with $3^{0}$ resolution of $\theta \& \varphi$. This results in 120 combinations for azimuth angle and 6 combinations for elevation angle for each TRM. Thus, each TRM can have 720(120x6) possible values of element phase.

For 576 elements, various possible combinations of element phase are 4,14,720 (720x576). MATLAB code is written to calculate the element phase of each element $(m, n)$ in the array for a specific value of elevation \& azimuth angles. The MATLAB output is generated in the form of Microsoft Excel file. The results include the element phase of all elements in the array for a specified beam position $(\theta, \varphi)$. The Excel sheets are produced using xlswrite function in MATLAB. The Table. 1 below is the MATLAB output produced in Excel sheet for $\theta=12^{0} \& \varphi=357^{0}$ with all combinations of $m$ \& $n$. But for simplicity only few combinations of $m \& n$ are taken. This Excel sheet is the output of MATLAB for calculated element phase.

\begin{tabular}{|c|c|c|c|c|c|c|c|c|c|c|c|c|c|}
\hline & -14 & -13 & -12 & -3 & -2 & -1 & 0 & 1 & 2 & 3 & 11 & 12 & 13 \\
\hline-14 & -694.12 & -641.80 & -589.47 & -18.58 & -66.25 & -13.93 & 38.39 & 90.71 & 143.03 & 195.35 & 613.93 & 666.25 & 718.57 \\
\hline-13 & -699.60 & -644.54 & -592.22 & -121.32 & -69.00 & -16.67 & 35.65 & 87.97 & 140.29 & 192.62 & 611.19 & 663.51 & 715.83 \\
\hline-12 & -699.60 & -647.28 & -594.96 & -124.06 & -71.74 & -19.42 & 32.90 & 85.23 & 137.55 & 189.87 & 608.95 & 660.77 & 713.09 \\
\hline-3 & -724.28 & -671.96 & -619.64 & -148.74 & -96.42 & -44.19 & 8.23 & 60.55 & 112.87 & 165.19 & 583.77 & 636.09 & 688.41 \\
\hline-2 & -727.02 & -674.70 & -622.38 & -151.48 & -99.16 & -46.84 & 5.84 & 51.81 & 110.13 & 162.45 & 581.03 & 633.35 & 685.67 \\
\hline-1 & -729.77 & -677.44 & -625.12 & -154.22 & -101.90 & -49.58 & 2.47 & 55.06 & 107.39 & 199.72 & 578.28 & 630.61 & 682.93 \\
\hline 0 & -723.51 & -680.19 & -627.86 & -156.97 & -104.64 & -52.32 & 0.00 & 52.32 & 104.64 & 156.97 & 575.28 & 627.86 & 680.19 \\
\hline 1 & -735.28 & -628.43 & -630.61 & -159.71 & -107.39 & -55.06 & -2.47 & 49.58 & 101.90 & 154.22 & 572.80 & 625.12 & 677.44 \\
\hline 2 & -737.99 & -685.67 & -633.35 & -162.45 & -110.13 & -57.81 & -5.48 & 46.84 & 99.19 & 151.48 & 570.06 & 622.38 & 674.70 \\
\hline 3 & -740.73 & -688.41 & -636.09 & -165.19 & -112.87 & -60.55 & -8.23 & 44.10 & 96.42 & 148.74 & 567.32 & 619.64 & 671.96 \\
\hline 11 & -762.67 & -710.35 & -656.03 & -187.13 & -134.81 & -82.48 & -30.16 & 22.16 & 74.48 & 136.80 & 545.38 & $\mathbf{5 9 7 . 7 0}$ & 650.02 \\
\hline 12 & -765.41 & -713.09 & -660.77 & -189.87 & -137.55 & -85.23 & -32.90 & 19.42 & 71.74 & 134.06 & 542.64 & 594.96 & 647.28 \\
\hline 13 & -768.15 & -715.83 & -663.51 & -192.61 & -140.29 & $\mathbf{- 8 7 . 9 7}$ & -35.65 & 16.67 & 69.00 & 121.32 & 539.89 & 592.22 & 644.54 \\
\hline
\end{tabular}

Table .1 The calculated element phase output produced using Excel Sheet for $\theta=12^{0} \& \varphi=357^{0}$ with $\mathrm{m}$ and $\mathrm{n}$ combinations. 


\subsection{Estimation of Absolute Phase Error}

The Phase shift control is achieved using a 6-bit digital phase shifter. The resolution of this phase shifter is $5.625^{\circ}$. Due to the quantization error of the digital phase shifter, the actual phase shift generated by each element may not be exactly same as the calculated phase shift. The difference between calculated element phase and actual element phase is estimated. This difference is the phase error caused due to quantization.For calculating the absolute phase error, the calculated elemental phase is rounded off to $5.625^{\circ}$ to get actual phase shift generated by the digital phase shifter. The calculated results are generated in the Excel sheets using MATLAB. The formula to calculate the absolute error is given as

\section{Absolute Error $=($ Calculated Element Phase $)-\quad$ (Actual Element Phase $)$}

\section{DEMONSTRATION OF THE ELEMENTAL PHASE}

The verification of calculated element phase was carried out using the test setup shown in Fig. 3 below:

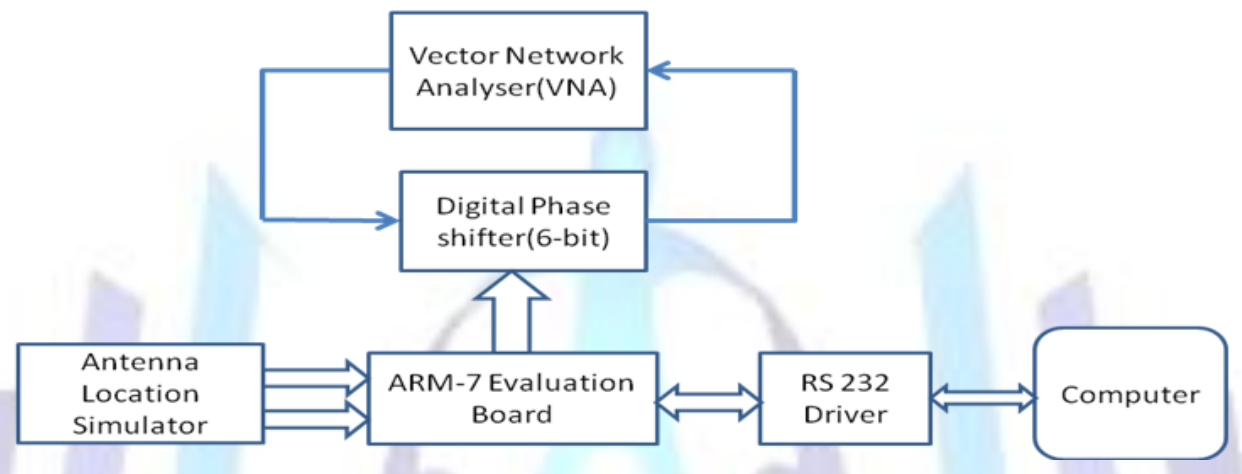

Fig. 3 Block Diagram of Elemental Phase Demonstration.

The set-up to check the element phase includes a 6-bit digital phase shifter is used in the TRM to generate the desired element phase. To demonstrate the element phase, this same 6 -bit phase- shifter is used. The insertion phase of this phase- shifter is measured by giving phase- shifter control word to generate $0^{0}$ phase shift. The measured insertion phase of the phase- shifter is compensated by generating appropriate control word and taking this control word as an offset for all element phase calculations. This ensures that calculated element phase and measured element phase match closely. The test set-up includes a Vector Network Analyser (VNA), which is used to measure the phase difference between input and output of the phase shifter. This phase difference gives the element phase for set values of $m, n, \theta \& \varphi$. The element phase is calculated using STM32F407VGT6 evaluation board [5] [6] [7].

For obtaining the $m$ and $n$ values, two 8- bit DIP switches are used. The microcontroller evaluation kit reads the values of $m \& n$ from the DIP switches. Out of this only 5 - bits are used, in which $5^{\text {th }}$ bit is used for assigning sign for $m$ and $n$. The $m$ and $n$ takes values in the range from -14 to 13 respectively. The 5 - bit is the most significant bit (MSB). The values of $m$ and $\mathrm{n}$ are obtained only when the respective bit switch is kept 'ON".

The values of $\theta \& \varphi$ are kept fixed and $m \& n$ are changed manually. The MCU STM32F407VGT6 calculates the element phase by reading $m \& n$ from one port and generates corresponding control word for phase shifter. The calculated element phase value is sent over USART to the PC through RS232 driver. The values of element phase calculated by MCU and measured at VNA are compared. The programming of STM32F4xx MCU was done in C using KEIL compiler (evaluation version) [8].The relative phase shift of the element is obtained by setting the appropriate control bits of the digital phase shifter.

For demonstration purpose this kit is being selected. The STM32F4xx is the ARM processor based on the ARMv7-M architecture [9] [10] [11] . Certain values of element phase (in each quadrant) were verified using the test set-up. All these elemental phase results are practically demonstrated. This results show significant change in the phase shift of the phase shifter.

This is a floating point processor which is basically used to calculate all the element phase calculations. This is the major advantage of this MCU. Floating-point calculations can be accelerated using a Floating-point unit (FPU) integrated in the processor [12]. On an FPU less processor, all these operations are done by software through the C compiler library and are not visible to the programmer; but the performances are very low. On a processor having an FPU, all of the operations are entirely done by hardware in a single cycle, for most of the instructions. The $\mathrm{C}$ compiler does not use its own floatingpoint library but directly generates FPU native instructions. When implementing a mathematical algorithm on a microprocessor having an FPU, the programmer does not have to choose between performance and development time. The FPU brings reliability allowing us to use directly any generated code through a high level tool, such as matlab or scilab. 
The 6- bit digital phase shifter will result into 64 positions i. e. $2^{6}=64$ positions. Therefore the resolution of the $6-$ bit phase shifter is $360^{\circ} / 64=5.625^{\circ}$. The LSB resolution is $5.625^{\circ}$ and MSB weight is $180^{\circ}$. The 6 - bit phase shifter provides a continuous beam steering of the main beam both in elevation and in azimuth angles in steps $3^{0}$. The MCU calculates the control word for digital phase shifter from the calculated value of element phase. When the control word corresponding to an element phase is output of STM32F407VGT6, the phase shift is changed, which is displayed on the VNA. The screen shot of Fig. 4 shows three trace windows (TR1, TR2, TR3). The TR1 window is the return loss (S11) of the phaseshifter which is $24.07 \mathrm{~dB}$ measured at $212 \mathrm{MHz}$. The TR2 window is the insertion loss (S21) which is $5.68 \mathrm{~dB}$, measured from $100-212 \mathrm{MHz}$ and linear throughout the frequency range. The TR3 window shows the output of the calculated element phase for $\theta=15^{\circ}$ and $\varphi=270^{\circ}$ is expected to be $33.75^{\circ}$ and the actual measurement output on the VNA is $33.92^{0}$.

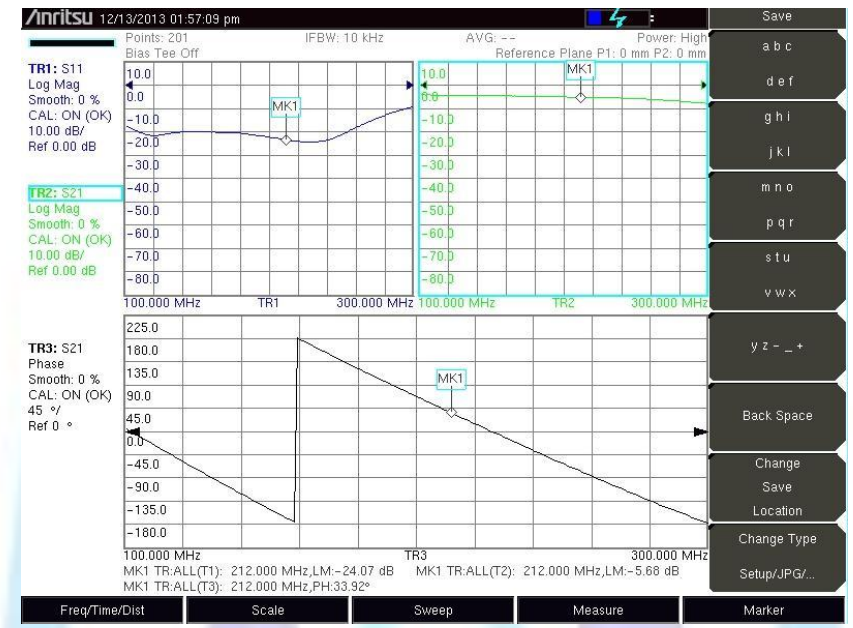

Fig. 4 output of the element phase on the Vector Network Analyser.

The Fig. 5 shows the actual set- up to demonstrate the element phase. The set -up comprises of the STM32F407VGT6 Discovery Evaluation Board, two 8 -bit switches and USART for serial communication with PC and Vector Network Analyser (VNA).

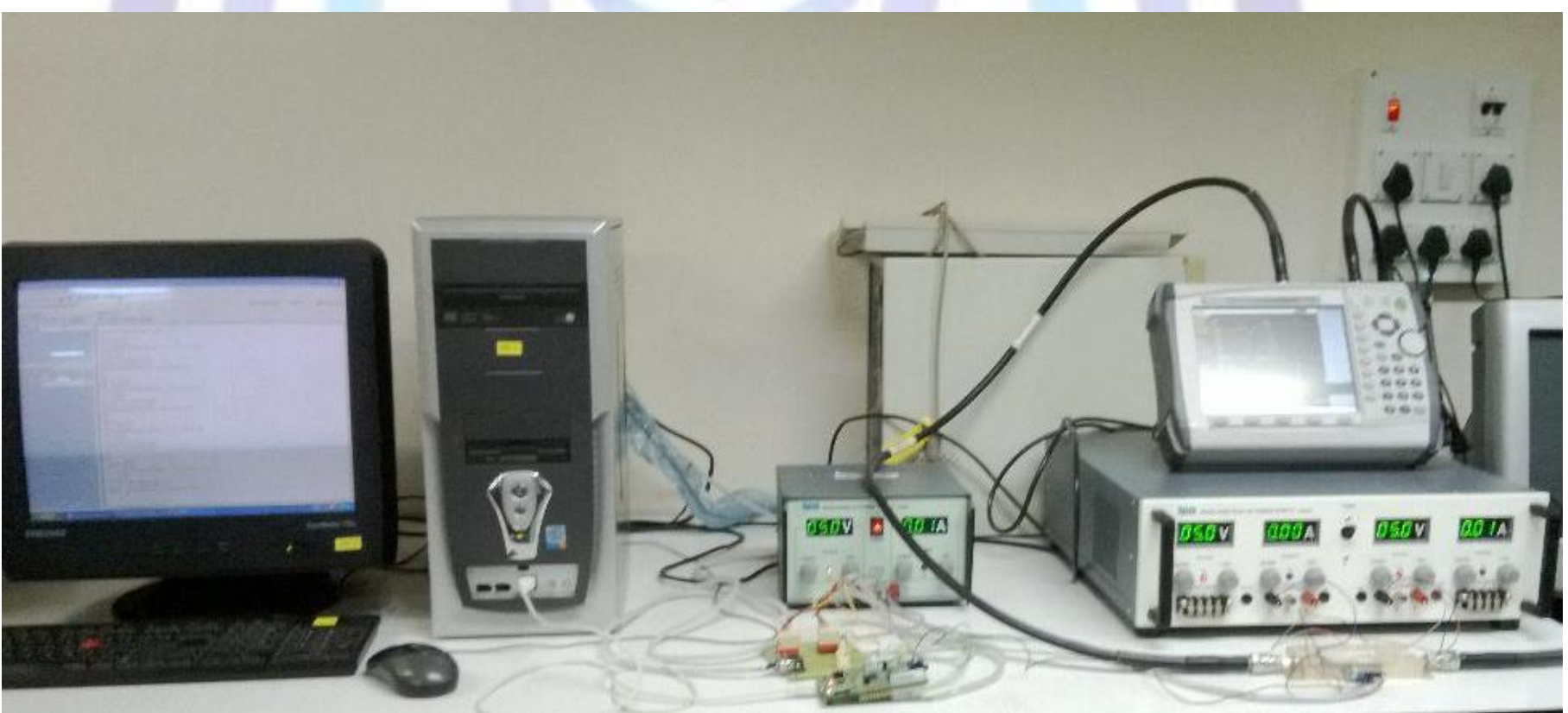

Fig. 5 Set-up to demonstrate the element phase. 


\section{CONCULSION}

The elemental phase calculation for each element in the antenna array for all beam positions has been carried out in MATLAB. The same were verified using a test set-up. The calculated element phase and measured element phase values match closely. The variation in calculated element phase and measured element phase is less than $2.8^{\circ}$, which indicates that the error is less than $1 / 2$ LSB of 6 - bit phase shifter. Also, absolute error between the calculated and actual elemental phase has been calculated for each element for all beam positions. The beam forming is fast and we can flexibly control the phase shift so that it forms the desired beam easily.

\section{ACKNOWLEDGEMENT}

The work was carried out at SAMEER (Society For Applied Microwave Electronics Engineering \& Research), IIT Bombay. The authors would also like to thank Mr. S.S. Kakatkar \& Mr. Bawanteinam Dkhar affiliated with SAMEER, Mumbai for their valuable comments and support.

\section{REFERENCES}

[1] "Microwave Theory of Phased - Array Antennas - A Review" Proceedings of the IEEE, VOL. 62, Dec. 1974, Louis Stark, Fellow IEEE, Invited Paper.

[2] Hansen R. C., 'Phased Array Antennas', WileBrooker, E. 'Practical Phased Array Antenna Systems', Arctech House.

[3] "Active Phased Array Antenna Based on DDS" L U Jiagno, East China Research Institute of Electronics Engg. P.R. China.

[4] "Basic Array Theory" Wolfgang H. Kummer, Life Fellow, IEEE, Invited Paper.

[5] "STM32F405xx/STM32F407xx ARM Cortex-M4 -Datasheet".

[6] "STM32F4 high-performance discovery board -STMicroelectronics".

[7] "Getting started with software and firmware environments for the STM32F4DISCOVERY Kit, User Manual".

[8] "STM32F3xxx and STM32F4xxx Cortex-M4 programming manual".

[9] "Discovery kit for STM32F407/417 line".

[10] "STM32 F4 series - High-performance Cortex-M4 MCU".

[11] "Reference manual STM32F40x, STM32F41x, STM32F42x, STM32F43x advanced ARM-based 32-bit MCUs".

[12] AN4044 -"Application note Using floating-point unit (FPU) with STM32F405/07xx and STM32F415/417xx microcontroller". 